\title{
Horizons/Théâtre
}

Revue d'études théâtrales

10-11 | 2017

Genre et arts vivants

\section{Angélica Liddell sur la scène française : des limites d'une transgression « féminine »}

\section{Ulysse Caillon}

\section{(2) OpenEdition}

\section{Journals}

\section{Édition électronique}

URL : http://journals.openedition.org/ht/522

DOI : $10.4000 /$ ht. 522

ISSN : 2678-5420

\section{Éditeur}

Presses universitaires de Bordeaux

\section{Édition imprimée}

Date de publication : 1 juillet 2017

Pagination : 114-135

ISSN : 2261-4591

\section{Référence électronique}

Ulysse Caillon, «Angélica Liddell sur la scène française : des limites d'une transgression « féminine » », Horizons/Théâtre [En ligne], 10-11 | 2017, mis en ligne le 01 juillet 2018, consulté le 19 juillet 2019. URL : http://journals.openedition.org/ht/522 ; DOI : 10.4000/ht.522

\section{(c) (i) $\odot$}

La revue Horizons/Théâtre est mise à disposition selon les termes de la Licence Creative Commons Attribution - Pas d'Utilisation Commerciale - Pas de Modification 4.0 International. 


\section{Ulysse CAillon}

Ancien élève de l'École Normale Supérieure de Paris et agrégé de Lettres Modernes, Ulysse Caillon est doctorant contractuel à l'université de Lyon 2 sous la direction d'Olivier Neveux, au sein du laboratoire IHRIM. Ses recherches portent sur les discours de l'intimité dans le théâtre européen contemporain (Pippo Delbono, Angélica Liddell, Fabrice Murgia, She She Pop...).

Mail : ulysse.caillon@gmail.com

Résumé: L'autrice et metteuse en scène espagnole, Angélica Liddell, a acquis une très forte visibilité sur la scène française ces dernières années. À partir de l'étude de trois de ses spectacles, cet article observe d'abord dans quelle mesure la parole y est donnée aux femmes, notamment autour des violences de genre. II s'intéresse ensuite à la subversion des normes de genre en rapprochant les images des spectacles de Liddell d'une esthétique queer et en s'interrogeant sur le traitement de la sexualité et de la violence. Enfin, il montre, en s'appuyant sur la

Abstract: Angélica Liddell, a Spanish playwright and director, has gained a great visibility in France over the past few years. Based on the study of three of her plays, this article first questions whether her theater makes women's voices heard, especially in the case of gender-based violence. It then analyses how gender norms are subverted in Liddell's shows, in images close to queer esthetics and through the treatment of sexuality and violence. Eventually, the article shows how this transgression of gender norms is thwarted by the artist's public statements on réception de la presse et sur le discours de l'artiste qui rejette le féminisme, les limites de la transgression des normes de genre dans ces spectacles. Son travail est alors envisagé comme réactivation d'une vision normée du féminin, à travers un discours sur l'amour et la douceur et un réinvestissement ambigu de la figure de l'hystérie.

Mots-clés : Angélica Liddell, théâtre de femmes, féminisme, sexualité, violences de genre

feminism and by press reviews. Her work can thus be viewed as a reinforcement of a normalized femininity, through images of love and care, and an ambiguous reenactment of hysteria.

Kerwords: Angélica Liddell, women's theater, feminism, sexuality, gender-based violence 


\section{Angélica Liddell sur la scène française : des limites d'une transgression « féminine »}

Ce n'est qu'en juillet 2010 que le public français a découvert Angélica Liddell, dont le travail était jusqu'alors diffusé principalement en Espagne. Invitée à présenter deux spectacles à l'occasion du Festival d'Avignon, L'Année de Richard (El Año de Ricardo) et La Maison de la force (La Casa de la fuerza), l'artiste est révélée surtout par le second, monstre de théâtre de cinq heures. Révélation qui devient rapidement adoption de la part du public, mais aussi de certaines institutions françaises : depuis 2010, Angélica Liddell est systématiquement invitée à présenter, voire à créer, ses nouveaux spectacles dans le circuit du théâtre public français, et notamment dans le cadre prestigieux du Théâtre de l'Odéon à Paris. Ce succès d'Angélica Liddell est à la fois surprenant et attendu. Une femme, de surcroît étrangère ${ }^{1}$, s'impose dans le milieu du théâtre public, grâce à un premier spectacle qui donne, comme on le verra, la parole aux femmes. Faut-il y voir un mouvement vers un théâtre qui laisse enfin la place aux femmes et qui pourrait déconstruire le mythe d'une différence naturelle des sexes ? Ou bien ce succès est-il le signe qu'au lieu de transgresser les normes de genre, le travail d'Angélica Liddell vient conforter des représentations et stéréotypes conformes à l'ordre de genre ? En analysant trois spectacles de Liddell, La Maison de la force, Tout le ciel au-dessus de la terre et You are my destiny, et en les confrontant à leur contexte de représentation, aux discours de l'autrice et à leur réception dans la presse, on s'attachera à éclairer ce cas particulier de la scène française.

\section{La parole donnée aux femmes}

\section{Combler le manque}

Il est particulièrement intéressant de constater que le succès d'Angélica Liddell en France, succès mesurable notamment par le nombre d'articles de presse qui lui sont consacrés, s'est produit au moment où les débats sur la place des femmes au théâtre prenaient de plus en plus d'importance. À la suite du rapport de Reine Prat au sein de la mission ÉgalitéS en 2006, promouvant « l'égal accès des hommes et des femmes aux postes de responsabilité, aux 
lieux de décision et à la maitrise de la représentation » dans le spectacle vivant ${ }^{2}$, les directeurs.trices de théâtres publics se trouvent confronté.e-s à la question de la parité dans leur programmation et incité.e.s notamment par des associations féministes à y inclure plus d'autrices et de metteuses en scène ${ }^{3}$. D'autre part, des spectacles collectifs féminins qui revendiquent leur féminisme reçoivent un très bon accueil à la même période. En 2012, Modèles de Pauline Bureau, joué au Rond-Point, dresse à travers les témoignages des comédiennes un tableau des oppressions sexistes contemporaines pour réaffirmer l'actualité des revendications féministes. Le Festival d'Automne à Paris accueille la même année pour la première fois She She Pop, « FrauenKollektiv » allemand, dont l'un des projets initiaux est de lutter contre l'hégémonie masculine dans l'écriture, la mise en scène et les institutions théâtrales ${ }^{4}$. On peut donc se demander si l'arrivée d'Angélica Liddell, qui a travaillé principalement avec des femmes, comédiennes professionnelles et amatrices, pour La Maison de la force, s'inscrit dans un mouvement plus global qui viserait à donner aux femmes plus de visibilité sur les scènes du théâtre public. Lors des saisons 2011-2012 et 2014-2015, elle est la seule femme dont un texte est joué à l'Odéon et également la seule metteuse en scène à assumer la direction non collective d'un spectacles. Caution féminine pour un théâtre souvent attaqué pour son manque de parité ? Son absence de la programmation 2012-2013 se traduit symboliquement par celle de toute autre autrice et metteuse en scène et a valu à l'Odéon une visite du collectif La Barbe lors de l'annonce de sa saison le 4 juin 2012. La fidélité de la scène nationale à l'artiste semble ainsi dissimuler une forme de nécessité, mais aussi un alibi avantageux pour l'institution, car Angélica Liddell est loin de revendiquer une position féministe, comme on le verra, et ne milite pas particulièrement pour une meilleure représentation des femmes dans les instances de la création théâtrale.

\section{Témoignages individuels et violence systémique : dénoncer le « paternalisme infect ${ }^{6}$ »}

Les spectacles de Liddell sont marqués par ce qu'elle appelle la « conscience d'être femme $\mathrm{e}^{7} \gg$. Ils mettent clairement la lumière sur une forme d'oppression produite par les hommes sur les femmes. La Maison de la force en est l'exemple le plus frappant. À l'orée du spectacle figurent deux « confessions » authentiques des comédiennes Getse de San Marcos et Lola Jiménez. La première relate une expérience de violence domestique, « raclée » que le compagnon juge «méritée », la seconde celle d'un viol's. Dans la deuxième partie du spectacle, A. Liddell débite un long monologue dans lequel elle raconte les 
humiliations et les violences domestiques commises par un ex-compagnon. Enfin, la troisième partie évoque les féminicides qui ont lieu dans la région de Chihuahua au Mexique, à travers les témoignages de trois autres femmes et de récits tirés d'articles de presse. La ville de Ciudad Juárez est en effet tristement célèbre pour les meurtres et les disparitions de femmes qui y ont lieu, et s'est retrouvée au cœur d'un débat public pour la reconnaissance institutionnelle du terme de « féminicide $\gg(\text { ou } \ll \text { fémicide } »)^{9}$. Des humiliations quotidiennes à la violence spectaculaire du féminicide, le spectacle met au jour une réalité, celle des violences de genre, qui devient son fil rouge. Le parallèle que le spectateur/la spectatrice est amenée à faire entre les témoignages individuels peut le mener à interpréter cette violence comme systémique. Ce partage d'expériences paraît alors dénoncer sans le nommer le patriarcat, structure d'oppression des hommes sur les femmes, dont les manifestations sont multiples.

En 2011, à Genève, où elle présentait Yo no soy bonita (Je ne suis pas jolie), Liddell disait ainsi vouloir lutter contre le « paternalisme $e^{10} \gg$ des hommes. Elle invitait à lire le titre de cette pièce, inspiré par une chanson populaire, comme une réaction à la réduction des femmes à leur physique ${ }^{11}$. Dans Tout le ciel au-dessus de la terre sous-titré « Le syndrome de Wendy », le spectacle s'attache à mettre en avant le personnage féminin occulté par Peter Pan, le héros éponyme du conte, comme contrepoint du célèbre syndrome de Peter Pan. Liddell donne la parole à Wendy, littéralement, et la fait sortir de l'ombre du héros masculin. Au point d'assumer elle-même dans ce rôle un monologue d'une heure vingt, couvrant plus de la moitié du spectacle. Ce sont donc les femmes qui parlent dans le théâtre de Liddell, ce que confirme encore une fois You are my destiny. Malgré un texte bien plus mince, le spectacle sous-titré «Le viol de Lucrèce » fait la part belle aux paroles de cette dernière, face à des figures muettes de Tarquin dont Liddell dit pourtant vouloir assumer le point de vue.

\section{Le syndrome d'Angélica}

Un second personnage féminin, identifié simplement par le pronom «Moi » dans le texte de la pièce, partage la parole avec Lucrèce dans You are my destiny. Sans surprise, c'est Liddell qui prend en charge ce texte face à son amant-fossoyeur. On entrevoit ici une première limite à une interprétation féministe du théâtre de Liddell, qui chercherait à donner la parole aux femmes. Dans ses spectacles, la voix de Liddell domine celle des autres. Ses monologues de La Maison de la force et de Tout le ciel au-dessus de la terre illustrent la disproportion dans la répartition du discours sur scène ${ }^{12}:$ davantage que 
des projets choraux, ces pièces s'inscrivent dans la « nébuleuse » du théâtre autobiographique ou autofictif ${ }^{13}$. Les spectacles comme les textes sont signés uniquement de son nom : son geste artistique ne cherche en rien à être collectif, comme chez Pauline Bureau ou She She Pop. Cependant, ces spectacles ne s'inspirent pas non plus uniquement de sa vie. En effet, elle s'accapare les histoires des autres au sein de projets souvent conçus comme proches de l'autobiographie.

De plus, si prendre soi-même la parole et s'exposer est un geste qui peut avoir une forte signification politique ${ }^{14}$, Liddell joue de sa singularité d'une façon qui détache son récit par moments de la question de l'occultation du discours des femmes. Liddell se définit à plusieurs reprises comme un « être inadapté », n'hésitant pas même à jouer avec la figure du « monstre », autant dans ses entretiens que dans ses texte ${ }^{15}$. Cette façon de se présenter fait de Liddell davantage une figure d'artiste isolée qu'elle ne l'inscrit dans un mouvement d'artistes femmes luttant pour leur reconnaissance dans le milieu artistique. La mise en avant continue de sa singularité est peu compatible avec le projet de donner collectivement la parole aux femmes, qui a pu être un axe de lecture de son travail dans un premier temps ${ }^{16}$, mais qui ne correspond pas à son projet artistique. Le travail de Liddell est donc ambigu vis-à-vis du féminisme : s'il contribue à faire entendre l'histoire, le quotidien du point de vue des femmes, il oppose à la collectivité la singularité d'une voix. Celle-ci, autocentrée dans sa lutte contre sa propre inadaptation, contrecarre un possible dévoilement de la violence du système patriarcal, évoquée parfois par Liddell sans qu'elle n'en fasse jamais le centre de son engagement artistique.

\section{Une transgression des rôles de genre?}

\section{Des images queer?}

Si l'on ne peut interpréter le théâtre de Liddell comme un théâtre dont le projet premier serait de donner la parole aux femmes, peut-on y lire cependant une entreprise de transgression des rôles genrés, qui remettrait en cause les repères du féminin et du masculin établis par les normes sociales ? Le théâtre de Liddell est à la fois très verbal et très visuel (You are my destiny fait figure d'exception parmi les trois spectacles, car il comporte beaucoup moins de texte, et notamment aucun monologue significatif de Liddell). Ces images se rapprochent d'une esthétique queer subversive, même si la pensée queer ou sa théoricienne la plus connue, Judith Butler, ne sont pas des références directes d'Angélica Liddell à notre connaissance. En effet, son théâtre trouble les représentations genrées, ce qui le rapproche d'une « praxis queer $»$, telle 
que définie par Elsa Dorlin comme «la mise en scène décalée, exubérante, parodique des normes dominantes en matière de sexe, de sexualité et de couleur $^{17} \gg$.

Dans les trois spectacles, elle s'amuse à arborer deux types de tenues qui surjouent la féminité : robes colorées et bouffantes d'une part, associées à un univers de conte de fées, tenues noires et moulantes de l'autre, signe d'une féminité plus sexuelle et menaçante. Liddell trouble ce surjeu de féminité par l'appropriation d'attributs masculins. Elle n'hésite pas, par exemple, à boire une quantité impressionnante de bière sur scène, à éructer, à côté d'un ballon de football dans You are my destiny. À la fin de la première partie de La Maison de la force, elle et deux autres comédiennes dénudent leurs torses, soulèvent des haltères, puis elles viennent prendre une pose plus lascive, à moitié allongées, les seins toujours nus, qui n'est pas sans évoquer la figure mythologique des Trois Grâces. La succession de ces deux images typiques de la masculinité et de la féminité met en évidence des codes sociaux ici subvertis. Les visions du féminin et du masculin sont dénaturalisées et envisagées comme constructions ou performances. Les comédiens qui incarnent Tarquin dans You are my destiny peuvent se travestir après avoir surjoué la masculinité, ou prendre des poses jugées féminines lorsque la chanson de Paul Anka qui a donné son titre au spectacle résonne à la fin de la pièce, tout comme Angélica Liddell et une autre comédienne peuvent endosser la masculinité de Harvey Keitel en rejouant une scène de Mélodie pour un tueur ${ }^{18}$ dans La Maison de la force, manipulant un pistolet imaginaire. Le genre des comédien'ne's n'est plus un critère absolu pour endosser des rôles féminins ou masculins : dans Tout le ciel au-dessus de la terre, c'est par exemple la voix d'une comédienne qui est celle du « policier », incarné simultanément par un comédien immobile. La dissociation entre le corps et la voix rappelle l'anti-essentialisme de la pensée queer ${ }^{19}$. D'autre part, Liddell compose des images qui sortent du cadre hétéronormatif, même si elle ne le remet pas explicitement en cause. Dans $\mathrm{La}$ Maison de la force, à la fin de la première partie, les comédiennes se caressent, s'embrassent, esquisses d'une sexualité lesbienne, tandis que les Tarquin nus de You are my destiny dansent en couples, convoquant subrepticement l'imaginaire gay ${ }^{20}$.

\section{La sexualité surexposée}

La sexualité est omniprésente dans l'univers de Liddell. On peut y lire une démarche subversive à nouveau, cette fois par le caractère frontal avec lequel le tabou de la sexualité féminine est abordé. Au début de Tout le ciel au-dessus de la terre, le public assiste à une scène de dix minutes de masturbation, exé- 
cutée par Liddell elle-même. Cette scène répond à la fois aux gestes codés de la masturbation féminine et les détourne, notamment lorsque Liddell frappe son bassin de manière répétée contre le sol, évoquant une pénétration masculine brutale, animée par des « coups de rein ». Liddell n'hésite pas à jouer avec les codes de l'érotisme que ce soit par les images ou par la parole. Dans You are my destiny, elle utilise la bière comme liquide érotique, s'en versant sur le corps, et lors du salut, elle jette sa culotte au public avant de parodier avec les autres comédien.ne-s une orgie sexuelle. Dans La Maison de la force, elle se lance dans un grand récit de ses errances sur un tchat pornographique où elle s'exhibe en webcam :

Et puis après, je me suis mise à tchatter, à me foutre à poil devant la webcam.

Je mettais $d u$ rouge à lèvres,

une jolie culotte,

un joli soutien-gorge,

et je branchais la caméra.

Eux, ils me montraient leur bite.

Moi je leur disais : « Ne me montre surtout pas ton visage, enfoiré, ne me montre pas ton visage.

Montre-moi juste ta bite. 》

Et moi non plus je ne montrais pas mon visage.

Et je leur disais de me demander des choses.

Ils me demandaient de me mettre un doigt dans le cul.

Et moi, je le faisais.

Ils me demandaient de me mettre un doigt dans la chatte.

Et moi, je le faisais.

Ils me demandaient de me pincer les tétons.

Et moi, je le faisais.

Ils me demandaient de me masturber.

Et moi, je le faisais.

Et je jouissais pour de bon.

Et certains me demandaient de me pisser dessus.

Mais moi, je n'arrivais pas à pisser.

$C^{\prime}$ 'st que moi, je ne sais pas pisser sur commande ${ }^{21}$.

Ce récit de la sexualité exhibée fait presque figure de manifeste de la part de Liddell qui n'hésite pas à définir sa démarche scénique comme une « pornographie de l'âme ${ }^{22} \gg$, dans une optique proche de ce que Patrice Pavis a qualifié de « confession impudique » en parlant du théâtre autobiographique $^{23}$. Liddell affirme à de multiples reprises que « rompre la barrière de la pudeur ${ }^{24} \gg$ est un de ses objectifs, ce qui passe par l'exhibition de son corps mais aussi celle de son «âme », en dévoilant sentiments et pensées les plus 
intimes, au-delà de ce qui est communément admis. Elle s'approprie alors pleinement les termes de cette exhibition : sexualité surexposée, mais contrôlée. « Où je veux, si je veux » peut-on lire entre les lignes de cette utilisation de la webcam, qui contrebalance le récit de Lola du début du spectacle, où la jeune femme ne pouvait mettre fin à un rapport sexuel devenu viol de plus en plus douloureux. Avec ironie, Liddell clame un peu plus loin une forme de droit à sa sexualité, « pute gratuite », conjurant le spectre d'une contrainte économique qui régirait cette exhibition, et s'écrie : «Je suis une bolchévique de la chatte. Je suis assez grande pour faire ma propre révolution sexuelle ${ }^{25}$. $\gg$

Dans Tout le ciel au-dessus de la terre, elle exprime ouvertement des désirs sexuels et tient tout un discours sur la masturbation, en écho à la scène d'ouverture :

Face à la maladie, face à la souffrance, il me vient juste une terrible envie de me masturber, de jouir, toute seule.

Quand je sors d'un hôpital, quand je viens de rendre visite à mon père à l'hôpital, je m'engouffre dans les toilettes d'un grand magasin pour me masturber, pour me faire jouir.

Ça me soulage.

Ça me fait passer la peur de la mort.

C'est ma façon de ressentir de la pitié pour tous ceux qui souffrent. Je me masturbe $e^{26}$.

Cette sexualité exhibée est d'autant plus transgressive que Liddell la met en regard d'événements qui, selon les normes sociales, ne doivent pas susciter le désir, comme ici la «maladie », la « souffrance ». La sexualité apparaît comme une réponse inattendue et inappropriée à des situations où l'on attend l'affliction, la retenue. Cette transgression va jusqu'à la provocation dans certains passages, où Liddell joue avec l'image psychiatrisante de la « dépravée sexuelle ${ }^{27} \gg$ : 
Quand je pense à la tuerie d'Utoya, je ne pense ni à la douleur, ni à l'horreur.

Quand je pense à la tuerie d'Utoya, je pense à tous ces jeunes gens que j'aurais aimés et qui ne m'auraient jamais aimée. J'imagine leurs sexes dans ma bouche. $J^{\prime}$ imagine d'éternelles fellations ${ }^{28}$.

Le discours de Liddell surexpose la sexualité féminine en l'appliquant à des situations inadaptées. Brandie de cette manière, elle détourne les codes de la pornographie classique, réappropriée par une femme loin du regard masculin, même si celui-ci est mis en scène dans le regard connecté du « pervers sexuel ». Ce jeu avec les codes de l'exhibition rapproche les spectacles d'Angélica Liddell du postporn dont quelques caractéristiques sont exposées ainsi par Rachele Borghi : « abolition de la distinction entre public et privé, usage de l'ironie, rupture avec la dichotomie sujet/objet, effacement de la frontière entre la culture légitime (l'art) et les productions culturelles illégitimes (la pornographie), implication des spectateurs, exposition publique de pratiques traditionnellement inscrites dans la sphère privée ${ }^{29} \gg$. Liddell réinvestit le discours de la sexualité et crée un récit dont elle est le sujet. La masturbation emblématise cette resubjectivation de la sexualité : sans homme qui ferait d'elle un objet potentiel, Liddell est maître de son désir, de son geste et de l'exhibition de celui-ci.

\section{La violence des femmes}

La sexualité est mêlée à la violence chez Liddell. D’une sexualité violente imposée par des hommes qui objectifient les femmes, on passe à une sexualité où les femmes sont sujets et s'adonnent elles-mêmes à une certaine violence. Liddell devient dominatrice sur les tchats sexuels dans Tout le ciel au-dessus de la terre:

Je suis très extrême, ils disent.

Il s'agit la plupart du temps de pratiques liées à l'humiliation. $[\ldots]$

A la fin, ils te demandent toujours si tu ferais ça pour de l'argent, si tu leur chierais dans la bouche pour de l'argent,

ils te supplient d'accepter,

ils t'implorent, ils sont prêts à payer.

Ils te demandent : « Tu n'aimerais pas avoir un esclave?» [...] 
Et c'est volontiers qu'ils se laissent humilier, car ils ne sont ni jeunes ni beaux mais vieux et répugnants, et ils deviennent plus soumis encore ${ }^{30}$.

Liddell revendique l'accès des femmes à la force par une appropriation transgressive de la violence. Exposant d'une part la violence sur les femmes, Liddell met aussi en scène une violence des femmes, sorte de « pouvoir des faibles $^{31} \gg$. Le discours de Liddell de La Maison de la force en est l'illustration : cette « maison de la force », « club de sport » où les haltérophiles sont pour la plupart des hommes, est l'endroit où Angélica vient se reconstruire après avoir subi les abus de son ex-compagnon, « raclées » physiques et verbales :

Je me suis entièrement consacrée à la surface.

Je me suis entièrement consacrée au monde de la force pour compenser ma faiblesse face au putain de monde de la force.

Je passais quatre heures par jour dans un club de sport.

Le seul moment où j'arrivais à être au calme, c'était dans la maison de la force, dans l'exercice de la force ${ }^{32}$.

Ce passage permet de lire la violence des femmes comme une vengeance contre celle subie précédemment de la part des hommes. Liddell dit s'identifier à Judith décapitant Holopherne ${ }^{33}$, faisant même directement référence à l'épisode biblique dans la pièce : «Après, quand j'avais fini de travailler mon cœur, je m'étirais comme Judith, / sauf que je n'avais pas une putain de tête au vestiaire ${ }^{34}$. »

La violence des femmes chez Liddell prend alors plusieurs formes, une violence des femmes contre les hommes; une violence des hommes que les femmes s'approprient ; une violence des femmes contre les femmes, ultime transgression d'un tabou. La première est la plus simple à lire. On en trouve une image frappante dans You are my destiny lorsque Liddell « torture » les Tarquin en les forçant à rester à l'équerre contre le fond de scène pendant une vingtaine de minutes ${ }^{35}$. Alors que ceux-ci crient d'épuisement, elle passe parmi eux en leur demandant de répéter après elle le dernier vers du Paradis de Dante : «L'amour qui meut le soleil et les autres étoiles ${ }^{36} \gg$, puis dépose une serpillère sur leur visage après avoir épongé leur sueur. Cette violence imposée aux comédiens offre une image retournée de la domination ${ }^{37}$, notamment celle de la violence domestique des hommes manipulateurs et pervers de $\mathrm{La}$ Maison de la force.

La deuxième forme de violence n'est plus dans le retournement, mais la réappropriation. Dans La Maison de la force, les trois comédiennes principales 
se livrent à des « exercices de bonheur pour fils de pute », où elles endossent le discours d'hommes d'une violence extrême :

LOLA - J'enfoncerai mon bras dans ton cul.

Je défoncerai ton putain de cul mort

avec mon bras.

Histoire de t'apprendre ce que c'est que l'amour.

Je vais te fissurer le cul en beauté

pour que tu arrêtes de geindre comme une sale gamine.

Tu vas savoir une bonne fois pour toutes ce que c'est, l'amour ${ }^{38}$.

ANGÉLICA - Embrasse-moi le cul.

C'est une phrase tout rose.

Embrasse mon putain de cul.

Rose cochon-heureux.

J'ai foutu en l'air la vie de plusieurs filles. Et alors?

Je les ai baisées, ensuite je les ai étranglées. Et alors ${ }^{39}$ ?

Ce retournement explicite des rôles n'est-il que témoignage de la violence des hommes ? Cette violence n'est-elle pas devenue celle des femmes ? Vociférante, hurlante, Liddell incarne une forme de violence absolue qui tend à dissoudre les rapports de genre au-delà d'une simple redistribution. C'est pourquoi sa violence finit par s'exercer contre tou.te.s, hommes, femmes, dominant.e.s et minorisé.e.s. Dans You are my destiny, après avoir torturé les Tarquin, elle crache sur Lola Jiménez, figure droite et immobile de Lucrèce, en l'insultant : « Fais quelque chose, pute ${ }^{40}$ ! \Son long monologue de Tout le ciel au-dessus de la terre déborde de violence verbale contre les mères avant de s'attaquer aux «infirmes $\gg$ :

Les mères, par exemple, les mères se sentent tellement fières des douleurs de l'accouchement qu'elles n'ont qu'une obsession: donner leur vie pour les autres.

J’ai donné ma vie pour toi !!!!

J'ai donné ma vie pour toi !!!!

Et puis quoi encore? Imbécile.

Pas besoin de ta sale vie.

Ta putain de vie, mets-la-toi dans ton cul.

[...] Dis-moi un peu, quel est ton supplément de dignité ?

D'après toi, qu'est-ce qui te rend meilleur que les autres?

Etre un infirme, ça te rend meilleur que les autres?

Soigner les infirmes, ça te rend meilleur que les autres?

Infirme égale gentil ? $\mathrm{Hein}^{41}$ ? 
Ce passage est d'autant plus violent que Liddell mime pendant qu'elle parle mères et infirmes, en s'en moquant. Elle fait éclater l'image de compassion associée au féminin et de la potentielle mère incarnée par chaque femme. La puissance subversive de la violence s'affirme encore davantage par l'usage de codes sadomasochistes dans certaines séquences. Dans le ballet érotique entre les comédiennes qui conclut la première séquence de La Maison de la force, l'une et l'autre se tirent les cheveux ou subissent les assauts d'un taser, dans un mélange de sensualité et de violence. On peut entrevoir une possible « faculté émancipatrice de la violence ${ }^{42}$ », même si elle est à relativiser, car il ne s'agit que d'un détournement dans ses représentations et non d'une appropriation révolutionnaire de la violence, comme ont pu la penser certaines féministes radicales ${ }^{43}$.

\section{Ambiguïtés et limites d'un discours « féminin »}

\section{« Yo no soy feminista » : la transgression artistique ne sera pas féministe}

«On dit toujours que la femme doit être féministe, mais moi je suis misogyne » affirmait Angélica Liddell lors d'un entretien à Montréal en mai 2014, autour de Tout le ciel au-dessus de la terre ${ }^{44}$. Si l'on a pu voir que la dramaturge-metteuse en scène jouait de sa position de femme, le féminisme n'entre pas dans son vocabulaire, voire en est exclu. « On me parle parfois de féminisme, mais, comme je l'ai déjà dit, je n'ai pas la sensation d'appartenir à un groupe, d'adhérer à une idéologie $\gg$ dit-elle encore à propos de son travail dans La Maison de la force ${ }^{45}$. « Idéologie », le féminisme est présenté ici comme une entrave à la liberté artistique. Afin d'affirmer une singularité absolue, Liddell préfère s'abstenir de cette étiquette et en brandir une autre, plus provocatrice et moins politiquement correcte, celle de «misogyne », même si elle ne se traduit pas par une esthétique particulière dans ses spectacles. Une partie du succès de Liddell est lié à sa capacité de provocation et à son apparent non-conformisme : You are my destiny, dans son projet, sa présentation et sa réception en est un exemple flagrant. Le spectacle a pour point de départ une violence de genre clairement identifiable, le « viol de Lucrèce $\gg$, qui en est aussi le sous-titre. Mais l'autrice de La Maison de la force change de point de vue en déclarant endosser celui de Tarquin, tandis qu'elle traite Lucrèce d' $\mathrm{d}^{\text {idiote }}{ }^{46}$ ». « Pourquoi ne pas pouvoir parler d'une femme qui tombe amoureuse de son violeur ? Pourquoi ne pourrait-on pas tisser une sorte de tissu que l'on tendrait au-dessus de l'enfer, et à travers lequel on pourrait le voir ? La poésie ne peut pas dépendre de l'idéologie ou de la justice » déclare Liddell citée par Fabienne Darge dans Le Monde. La journaliste 
souligne : «C'est une chose qui a toutes les chances d'être mal comprise ${ }^{47}$, par les temps qui courent $t^{48}$. $\gg$ De son côté, la présentation du spectacle sur le site de l'Odéon valorise ce retournement et la position singulière de Liddell :

Certains auraient pu s'attendre à ce qu'Angélica Liddell propose une relecture féministe radicale de la légende. Il n'en est rien. Loin de s'attacher à réinterpréter la souffrance de Lucrèce, c'est la souffrance de Tarquin qu'elle aborde - autrement dit, celle d'un homme. Au lieu d'opposer à une simplification politique une contre-simplification qui resterait finalement enfermée sur le même terrain, Liddell invente un tout autre plan. Son intention n'est pas, n'a jamais été, de corriger ou supplanter un point de vue masculin par un point de vue féminin ${ }^{49}$.

Fabienne Pascaud renchérit dans Télérama : «On ne peut reprocher à la performeuse de 48 ans d'être artistiquement correcte et dans les clous d'un féminisme conventionnel ${ }^{50}$. $\gg$ Le féminisme se voit donc renvoyé du côté de la $\ll$ convention $\gg$, du « correct $\gg$, de la pensée majoritaire des « temps qui courent $\gg$. Il devient une norme à éviter pour un théâtre singulier, provocateur et subversif, capable de briser les horizons d'attente. On peut noter un écart étonnant entre les déclarations que Liddell faisait au moment de $\mathrm{La}$ Maison de la force où le féminisme était simplement tenu à distance, et celles concernant You are my destiny, où il est clairement repoussoir. « Je ne supporte pas, dit-elle, ce règne du féminisme qui ne cesse de culpabiliser les hommes. J'ai voulu voir l'homme dans sa fragilité à l'instant où on le voit dans la situation du bourreau ${ }^{51}$. » Mais n'est-ce pas Liddell qui véhicule elle-même une image conventionnelle et bien peu subversive du féminisme comme haine des hommes ? Le refus de l'étiquette « féministe » ou « femme » traduit une volonté d'être perçue avant tout comme artiste et « poète » auprès du public $^{52}$. La question du féminisme ou de l'antiféminisme devient alors pour elle « absurde $»$, car « la poésie n'est pas la justice », déclare-t-elle ${ }^{53}$, comme si elle cherchait à transgresser des normes artistiques aux dépens de normes sociales. Ce refus s'inscrit alors dans celui d'un théâtre trop explicitement politique qui perdrait sa noblesse et sa capacité d'innovation à se faire militant, attaque qu'Olivier Neveux relève dans son introduction à Théâtres en lutte : «La littérature militante se voit reprocher pêle-mêle ses schématismes, ses simplismes, son peu d'ambition formelle ; on lui refuse, régulièrement, toute intelligence artistique, voire toute intelligence politique ; on lui oppose un art formaliste, expérimental, noble ${ }^{54}$. 》 


\section{« L'amour qu'une femme ressent pour un homme »}

Ce rejet clair du féminisme chez Liddell à partir de You are my destiny est marqué par un discours parallèle à celui de la violence, qui met en avant son opposé, la «douceur » et l'« amour ». Les mots qui concluent la pièce sont à cet égard révélateurs :

Et voilà comment un violeur fit de moi son amante. Car, de tous les hommes qui m'entouraient, père, époux et ami, fanatiques de ma vertu, esclaves de leurs ambitions, avec sur leur couteau mon sang encore chaud, le seul qui ait parlé d'amour, le seul qui n'ait pas parlé de patrie, le seul qui n'ait pas parlé de gouvernement, le seul qui n'ait pas parlé de guerre, le seul qui n'ait pas parlé de politique, le seul qui ait préféré tout perdre en échange d'un instant d'amour, c'est le violeur, c'est Tarquin ${ }^{55}$.

Contrairement à la violence et à la sexualité, l'amour n'est pas présenté comme une construction sociale, un discours, mais comme un mystère qui ne peut être analysé. Opposé à la « patrie », la « politique », l'amour devient une force naturelle, non seulement dans You are my destiny, mais aussi dans tout l'univers de Liddell. La Maison de la force s'achève sur le cri de « L'homme fort », haltérophile de plus de cent kilos, déclamant : « Aimer à ce point pour mourir si seuls ${ }^{56} ! \gg$ La pièce gravite autour du thème du manque d'amour. Au milieu des tchats sexuels, Liddell injecte la question de l'amour :

Quand tu n'as pas d'amour véritable, tu prends

n'importe quelle connerie

comme un succédané d'amour.

C'est comme si on t'aimait.

Ça m'est égal qu'ils soient gros, vieux, sales, sales, sales, sales ou moches.

Ça m'est égal.

Si je ne peux pas être aimée,

ça m'est égal ${ }^{57}$.

Compensant le caractère cru de la sexualité, l'amour convoqué par Liddell, renvoie à une forme de « douceur » qui atténue la violence des situations présentées et rend plus ambiguë la réappropriation qu'elle en fait. Parmi les commentaires de la presse sur You are my destiny, beaucoup insistent sur cet aspect, comme celui de Jean-Pierre Thibaudat, pour qui le spectacle est « traversé de bout en bout par l'amour » et d'une « douceur » qui « canalise la violence $^{58} \gg$. De même Annie Chénieux évoque la « douceur » qui « imprègne » le spectacle en réponse à la « douleur » exposée de la metteuse en scène ${ }^{59}$. Cette insistance sur la douceur dans la presse souligne l'ambiguïté du travail de Liddell, qui d'un côté sape l'imaginaire d'une douceur féminine 
par une mise en scène de la violence des femmes et de l'autre le ravive. Car la douceur reste un idéal à atteindre, si l'on en croit un extrait du monologue de Tout le ciel au-dessus de la terre: « Je me rends compte que j’ai seulement la capacité d'aimer. / L'amour. L'amour. / C'est mon unique sentiment. / Je parle de l'amour qu'une femme ressent pour un homme ${ }^{60}$. $\gg$

Cette rengaine des textes de Liddell sur le besoin et le manque d'amour, et l'appel à une forme de douceur nourrissent finalement l'idéal d'une complémentarité entre hommes et femmes. L'indépendance des figures féminines sur scène s'avère fragile et souvent contrainte. Wendy emblématise cette position : « abandonnée » par Peter Pan, elle est à la recherche de l'amour perdu et d'un homme qui pourra le remplacer. De même, la fin de You are my destiny est marquée par l'image du couple formé par Liddell et le « Fossoyeur » d'un côté, de l'autre par Lucrèce (Lola Jiménez) et un des Tarquin, tous deux dénudés et recouverts de feuilles, évocation du couple biblique d'Adam et Eve. Cette référence picturale à l'Eden érige le couple hétérosexuel comme une sorte de modèle éternel, ce qui se lit dans la formule « Je parle de l'amour qu'une femme ressent pour un homme. $\gg$ Si l'exposition des violences de genre peut servir à dénoncer un système patriarcal, l'hétérosexualité ellemême n'est pas interrogée et apparaît comme modèle naturel. Alors que quelques images queer bousculent les normes de genre dans la dramaturgie de Liddell, cette naturalisation de l'hétérosexualité, dans un mouvement inverse, les conforte, sapant en partie le potentiel subversif des spectacles.

\section{Réactiver les clichés du féminin}

Dans l'introduction à leur ouvrage Penser la violence des femmes, Colette Cardi et Geneviève Pruvost remarquent que les représentations de la violence féminine répondent à un « double mouvement, en apparence paradoxal, qui, d'un côté, fait de la violence du sexe faible un tabou, passant sous silence des pratiques pourtant récurrentes, ou qui, de l'autre, hypertrophie cette violence pour en stigmatiser la démesure ${ }^{61} \gg$. La violence des femmes qui vient briser une forme de tabou, imposé par des normes de genre, pourrait être plus ambiguë que ce que nous avons exposé précédemment. En effet, Liddell n'estelle pas souvent dans l'« hypertrophie », la « démesure » ? Les cris, vociférations, trépignements récurrents dans son attitude correspondent aussi à une image normée, contrepoint de la douceur féminine évoquée. Si l'on reprend l'exemple de You are my destiny, la presse ne peine pas à trouver des figures féminines classiques pour caractériser cette violence scénique. Odile Quirot parle d'elle comme de « cette brindille à l'œil noir, qui pythie et poétesse, n'a pas la langue dans sa poche ${ }^{62} \gg$ tandis que pour Jean-Pierre Léonardini, elle 
« se conduit en sorcière (bruja en espagnol), en hechicera (magicienne), en ménade de la horde dionysiaque menant le bal échevelé final ${ }^{63} \gg$. Le biais du genre est également tout à fait sensible dans les adjectifs employés : « excessive, narcissique, mystique ${ }^{64} \gg$ ou $\ll$ furieuse, rageuse, ravageuse ${ }^{65} \gg$. Angélica Liddell ravive le cliché de la femme blessée et vengeresse avec des spectacles pétris de sentiments de rage et colère, à l'opposé du calme froid de la raison. Son univers est donc naturellement celui de l'« hystérie de l'instant ${ }^{66} \gg$. Le théâtre de Liddell joue avec l'hystérie, mais jamais de façon explicite, ce qui revient encore une fois à naturaliser une vision normée du féminin. Les excès dans l'attitude de Liddell, démesure de la parole, violence physique et verbale, mais aussi surexposition de la sexualité, forment un tableau clinique de la féminité malade, influencé par de multiples représentations genrées. « Sorcière $\gg$ de la scène, Liddell ne dénonce pas ces clichés du féminin, ne les expose pas comme matériau à déconstruire, et ainsi les réactive. Les critiques de la presse traduisent ce manque de distance, ne montrant elles-mêmes aucun second degré par rapport aux images de « pythie » ou de « sorcière ». Féminité excessive, mise à l'écart, et même exotisée, dans les termes espagnols employés par J.-P. Léonardini ${ }^{67}$ : si Angélica Liddell ne colle pas à certaines images sociales du féminin, elle se glisse parfaitement dans d'autres codes, ceux de l'hystérie et de la démesure pathologique, tout autant régis par un partage genré.

Angélica Liddell occupe une place ambiguë sur la scène française. Révélée par un spectacle, La Maison de la force qui mettait fortement en exergue les violences de genre et leur caractère systémique, elle a représenté pour le théâtre français l'opportunité de mettre en avant une femme autrice, metteuse en scène et actrice, dont le discours, en mettant plus ou moins subtilement à distance le féminisme, était marqué malgré tout par la revendication d'être femme. En outre, ses spectacles mettent au travail les normes de genre, en produisant des images éloignées des représentations quotidiennes de la féminité et de la masculinité, ce qui contribue à faire apparaître le masculin et le féminin comme des objets construits, avec lesquels il est possible de jouer. Liddell reconnaît cependant elle-même s'être écartée progressivement d'une approche « matérialiste » dans son travail pour privilégier « l'angoisse mystique $^{68} \gg$, annonçant dans le prologue de You are my destiny un passage au « temps du sacréé ${ }^{9} \gg$. Cette évolution met en lumière des tendances déjà à l'œuvre dans son travail : celle d'une naturalisation du féminin par l'absence 
de remise en cause de l'hétérosexualité, qui réactive le mythe d'une complémentarité entre hommes et femmes ; et par une image de féminité excessive proche de l'image normée et pathologique de l'hystérie. En exposant un « Je » surdimensionné, Liddell réactive l'idée d'une féminité incontrôlée, à l'écart d'une sphère masculine de la rationalité. On ne peut donc faire du travail de Liddell une entreprise univoque de transgression des normes de genre, et ce n'est sûrement pas là l'effet recherché par l'artiste, qui, au nom d'une volonté de subversion, rejette toute adhésion à une «idéologie », afin d'affirmer sa liberté artistique et poétique. Cette dissociation revendiquée entre l'art, la morale et la politique ${ }^{70}$ - qui va à l'encontre d'une tendance contemporaine des artistes à réaffirmer toujours plus le caractère politique du théâtre ${ }^{71}$ - crée un public scindé entre deux horizons, qui ont bien, eux des enjeux politiques : l'un qui espère encore de la part d'Angélica Liddell une éclosion féministe, à partir des graines prometteuses semées dans son théâtre, l'autre qui se voit conforté et rassuré que le féminisme soit maintenu comme repoussoir par une artiste femme.

\section{Notes}

1. On peut en réalité penser qu'en tant qu'étrangère, statut d'exception, Angélica Liddell accède plus facilement aux institutions nationales prestigieuses que d'autres artistes femmes de théâtre françaises.

2. Reine Prat, «Mission pour l'égalité et contre les exclusions - rapport d'étape $n^{\circ} 1$ » [en ligne], Ministère de la culture et de la communication, mai 2006, http://www.culture. gouv.fr/culture/actualites/rapports/prat/egalites.pdf, consulté le $1^{\text {er }}$ avril 2016.

3. Voir Lorraine Wiss, «Les enjeux de la parité dans le spectacle vivant », Théâtre/Public $n^{\circ} 224$, avril-juin 2017, p. 30-38.

4. Cf. Sonja Kloevekorn, "Leben im Kollektiv » [en ligne], Deutschland Radio Kultur, 8 mars 2012, http://www.deutschlandradiokultur.de/leben-im-kollektiv.1153.de.html?dram:article_id=182688, consulté le $1^{\text {er }}$ avril 2016. L'article décrit le parcours de Mieke Matzke, fondatrice de She She Pop, qui alors étudiante à l'université de Gießen en études théâtrales, " pour protester mais aussi s'amuser " (" aus Protest und auch aus Spaß ») contre " un partage classique des rôles parmi les étudiants » (" eine klassische Rollenverteilung unter den Studierenden »), crée avec sept "camarades militantes " ("Kommilitoninnen») un " collectif de femmes " ( Frauenkollektiv»).

5. En 2013-2014, elle et Katie Mitchell sont les seules metteuses en scène de la saison, tandis que sont joués trois textes écrits par des femmes : celui d'Angélica Liddell, un de Charlotte Perkins Gilman monté par Katie Mitchell, le dernier de Catherine Anne montée par Joël Pommerat.

6. «Il faudrait vivre en dehors du monde ou être stupide pour ne pas se rendre compte de la position occupée par les femmes dans notre société, qui est une société parfaitement misogyne, où règne un paternalisme infect, même si parfois c'est à peine perceptible ", 
Angélica Liddell sur la scène française : des limites d'une transgression « féminine »

entretien avec Christilla Vasserot le 26 janvier 2011, dans Outre scène n ${ }^{\circ}$ 12, mai 2011, p. 17.

7. Ibid.

8. «Un jour, je suis avec un gars, je ne le connais pas, on est en train de baiser, et lui, il commence à me baiser violemment, il me fait mal, TRÈS mal, alors moi je pleure, je lui dis d'arrêter, mais lui, il ne s'arrête pas, il continue à me baiser et moi je continue à pleurer, je suis incapable de lui dire de partir », La Maison de la force, trad. par Christilla Vasserot, Besançon, Les Solitaires Intempestifs, 2012, p. 62-63.

9. Voir Chiara Calzolaio, «Les féminicides de Ciudad Juárez : reconnaissance institutionnelle, enjeux politiques et moraux de la prise en charge des victimes ", Problèmes d'Amérique latine $\mathrm{n}^{\circ}$ 84, 2012, p. 61-76. En 2007, un film grand public, Les Oubliées de Juarez (Bordertown), portait également sur le thème des féminicides dans la région, illustrant l'ampleur de son écho médiatique.

10. Entretien à la Bâtie, Festival de Genève 2011, disponible en ligne : http://vimeo. com/29018891, consulté le 24 juillet 2017.

11. "Je ne suis pas jolie et je ne veux pas l'être [Yo no soy bonita y no quiero ser]". Ce sont les paroles d'une chanson. Cette phrase a à voir avec le paternalisme. [...] Alors le marin dit : "comme tu es belle, tu ne paies rien". Moi je veux payer. Je ne veux pas quon me laisse passer parce que je suis belle. C'est le paternalisme, c'est le loup, c'est le monstre... je préfère ne pas être belle ", ibid.

12. Dans Tout le ciel au-dessus de la terre, trad. par Christilla Vasserot, Besançon, Les Solitaires Intempestifs, 2013, 38 pages sur les 73 du texte, correspondant à une heure vingt de monologue sur deux heures et quart de spectacle ; dans la deuxième partie de La Maison de la force, 17 pages sur les 36 du texte, correspondant à trente-cinq minutes sur une heure trente-cinq minutes de spectacle.

13. Voir Florence Fix et Frédérique Toudoire-Surlapierre (dir.), L'Autofiguration dans le théâtre contemporain, Dijon, EUD, 2011. Le terme de " nébuleuse » est emprunté à Vincent Colonna qui l'utilise pour parler de l'autofiction dans le domaine littéraire, voir Vincent Colonna, Autofiction et autres mythomanies littéraires, Auch, Tristram, 2004, p. 11.

14. Deirdre Heddon interprète ainsi un certain théâtre autobiographique comme relevant d'une " praxis féministe ", au sens où il reprend l'idée contenue dans la bannière féministe " le privé est politique ", dans Deirdre Heddon, Autobiography and Performance, Hampshire, Palgrave Macmillan, 2008, p. 23.

15. Voir par exemple dans l'entretien à La Bâtie en 2011 : «J'ai toujours parlé des monstres parce que je me considère comme un être taré [tarado], un être inadapté à la vie sociale », dans Tout le ciel au-dessus de la terre, op. cit., p. 59 : "Je sais que je suis un monstre."

16. Cette approche est par exemple sensible dans le dossier d'accompagnement pédagogique élaboré par l'Odéon pour La Casa de la Fuerza, qui, dans une partie intitulée "Le contexte ", propose de mettre en relation la pièce avec des statistiques de l'observatoire de la parité entre les hommes et les femmes. Disponible en ligne : http://www. theatre-odeon.eu/sites/default/files/da_import/file_823_DA_La-casa.pdf, consulté le 24 juillet 2017.

17. Elsa Dorlin, Sexe, genre et sexualités, Paris, PUF, 2008, p. 111. 
18. Mélodie pour un tueur, James Toback, 1978. La référence au film est explicite dans le spectacle et le texte de Liddell, La Maison de la Force, op. cit., p. 65-66.

19. «Sur le plan normatif, les auteurs queer ont promu une politique anti-essentialiste, formulant des revendications sur le terrain de l'identité mais en considérant en même temps que celle-ci n'est pas stable, et que sa construction ne répond qu'à des considérations stratégiques. Selon les mots de David Halperin, il s'agit d'une "identité désessentialisée et purement positionnelle" qui "prend son sens dans sa relation d'opposition à la norme" [Saint Foucault, Paris, EPEL, 2000 [1995], p. 75]. Plutôt que de revendiquer une identité sur la base d'une homosexualité positivement pensée comme un choix d'objet, les stratégies politiques queer placent en leur cœur une identité d'opposition et de combat, une identité "sans essence" qui est mobilisée par rapport à et contre la norme dominante ", Laure Bereni, Sébastien Chauvin, Alexandre Jaunait et al., Introduction aux études sur le genre, $2^{\mathrm{e}}$ édition revue et augmentée, Louvain-la-Neuve, De Boeck, 2012, p. 51.

20. Dans la conception de La Maison de la force, dont le titre fait référence à la salle de sport, que Liddell fréquente lorsqu'elle écrit le journal intime qui sert au spectacle, elle dit avoir été marquée par les corps des acteurs pornos gays qu'elle y croisait. (« [...] mi gimnasio está lleno de actores porno gays, alegres y guapos, que desean amar y ser amados, como todos nosotros " : texte publié dans l'édition espagnole de la pièce, La Casa de la Fuerza, Segovie, La uÑa RoTa, 2011, page intérieure de couverture).

21. La Maison de la force, op. cit., p. 79.

22. Cette expression, récurrente chez Liddell, est par exemple présente dans son entretien avec Christilla Vasserot lors du 64 Festival d'Avignon : «Dans La Maison de la force, $[. .$.$] j'ai travaillé avec la douleur. Il n'y a pas de médiation, pas de personnage$ [...] qui fasse office de médiateur. C'est la pornographie de l'âme, une pornographie spirituelle ", disponible en ligne : http://www.festival-avignon.com/lib_php/download. php?fileID=95\&type=File\&round=84586581, consulté le 11 octobre 2017.

23. Patrice Pavis, "Théâtre autobiographique ", Dictionnaire du théâtre, Paris, Dunod, 1996, p. 361-362. Voir également Louis-Patrick Leroux, « Théâtre autobiographique : quelques notions ", Jeu n ${ }^{\circ} 111,2004$, p. 75-85.

24. Par exemple dans l'entretien précédemment cité avec Christilla Vasserot pour le $64^{\mathrm{e}}$ Festival d'Avignon.

25. La Maison de la force, op. cit., p. 80.

26. Tout le ciel au-dessus de la terre, op. cit., p. 63.

27. Ibid., p. 64.

28. Ibid.

29. Rachele Borghi, « Post-porn », Rue Descartes n 79, 2013, p. 29.

30. Tout le ciel au-dessus de la terre, op. cit., p. 67-68.

31. «Malgré tout, dans la violence de certaines femmes qui n'interrogent pas pour autant la question de leur sexe, semblent subsister des stigmates de ce que l'on pourrait appeler le pouvoir des faibles ", Arlette Farge, préface à Coline Cardi et Geneviève Pruvost (dir.), Penser la violence des femmes, Paris, La Découverte, 2012, p. 11.

32. La Maison de la force, op. cit., p. 78. 
Angélica Liddell sur la scène française : des limites d'une transgression « féminine »

33. «Cela dit, s'il est une icône avec laquelle je m’identifie vraiment, c'est Judith. Judith décapitant Holopherne. C'est peut-être l'image qui m'a le plus influencée », entretien avec Christilla Vasserot dans Outre scène, op. cit., p. 24.

34. La Maison de la force, op. cit., p. 78-79.

35. Fabienne Pascaud dans Télérama décrit ainsi cette séquence : "Quand l'actrice-auteur-scénographe-metteuse en scène a fini de régler leur compte à dix machos à poil sur le plateau rouge sang, soudain réduits à rien devant un palais des Doges vénitien de carton-pâte », dans « Entre douleur et plaisir, les tortures scéniques d'Angélica Liddell » [en ligne], Télérama, 8 décembre 2014, http://www.telerama.fr/sortir/entre-douleuret-plaisir-les-tortures-sceniques-d-angelica-liddell,119894.php, consulté le $1^{\text {er }}$ avril 2016. Lexpression " régler leur compte " associée à la qualification de " machos » des Tarquin exprime bien l'idée d'une violence « vengeresse » retournée contre les hommes.

36. "L'amore che move il sole e l'altre stelle », en italien dans le spectacle, phrase non reprise dans le texte publié en français.

37. Fabienne Darge écrit ainsi que Liddell " règne sur les hommes " à cette occasion. Fabienne Darge, «Théâtre : sacrée Angélica Liddell » [en ligne], Le Monde, 3 décembre 2014, http://abonnes.lemonde.fr/scenes/article/2014/12/03/theatre-sacree-angelica-liddell_4533624_1654999.html, consulté le $1^{\text {er }}$ avril 2016.

38. La Maison de la force, op. cit., p. 86.

39. Ibid., p. 88.

40. «Fa' qualcosa, puttana! » réplique en italien dans le spectacle, non reprise dans le texte publié.

41. Tout le ciel au-dessus de la terre, op. cit., p. 61-62.

42. Arlette Farge, préface à Coline Cardi et Geneviève Pruvost (dir.), Penser la violence des femmes, op. cit., p. 12.

43. On pense par exemple au SCUM Manifesto de Valérie Solanas et à l'appel de Florence Rochefort à « sortir les couteaux » dans la préface à sa traduction française. Mais en réalité, comme le notent Coline Cardi et Geneviève Pruvost, la violence est peu revendiquée comme moyen d'action par les groupes d'action féministe, " considérée [...] comme un attribut patriarcal qui doit être condamné sous toutes ses formes ", dans "Introduction générale » à Penser la violence des femmes, op. cit., p. 27. Seules quelques actions sont relevées par les autrices, notamment celles des suffragettes anglaises avant 1914 ou l'attaque au mou de veau du professeur Lejeune en 1971. Le recours à la violence se fait davantage dans " des mises en scènes parodiques et insolentes sans passage à l'acte, privilégiant la performativité de la représentation sur la violence effective : ce sont les torchons qui brûlent et non les maris qui sont immolés ", ibid., p. 44.

44. Entretien avec Philippe Couture, disponible sur le site theatre-contemporain.net, http://www.theatre-contemporain.net/spectacles/Todo-el-cielo-sobre-la-tierra-Elsindrome-de-Wendy-7889/entretiens/idcontent/50201, consulté le $1^{\text {er }}$ avril 2016.

45. Entretien avec Christilla Vasserot, lors du 64e festival d'Avignon, précédemment cité.

46. "Quand je pense au poème du viol de Lucrèce, Lucrèce me semble être une idiote. » [ "Cuando me enfrento al poema de la violacion de Lucrecia, Lucrecia me parece una idiota. »] Entretien avec Jordi Batallé, « Angélica Liddell : Hay que restaurar el espíritu de lo sagrado » [en ligne], RFI, 12 décembre 2014, http://es.rfi.fr/cultura/20150805-angelica-liddell-hay-que-restaurar-el-espiritu-de-lo-sagrado, consulté le 11 octobre 2017. 
47. Liddell semble être en effet régulièrement « mal comprise », et cela même par Fabienne Darge. Marion Siéfert analyse ainsi le cas exemplaire de la réception de You are my destiny dans un article intitulé "Tirez sur la sorcière ", dans Agôn, Critiques, Saison 2014-2015, disponible en ligne : http://agon.ens-lyon.fr/index.php?id=3166, consulté le 24 juillet 2017. «Dans les reproches qu'ils adressent à You Are My Destiny, les critiques opèrent par réduction : ils réduisent le spectacle à un pitch provocateur (Liddell transforme un viol en une histoire d'amour) et balaient ainsi d'un revers de main l'ensemble de son œuvre précédente qui, de Mais comme elle ne pourrissait pas... Blanche-Neige à La Casa de la Fuerza, traitait avec une profondeur et une finesse rarement rencontrées la question du viol. Peut-on honnêtement croire que la metteuse en scène aurait aussi promptement retourné sa veste pour défendre cette fois-ci le violeur ? C'est pourtant la thèse que nombre de critiques semblent soutenir. Tous la rappellent à l'ordre et condamnent sévèrement son manque de morale et son inconscience politique. » Cette unanimité de la critique est cependant à nuancer (voir la suite de l'article).

48. Fabienne Darge, Le Monde, art. cit.

49. Texte de présentation rédigé par Daniel Loayza, dramaturge du théâtre de l'Odéon, disponible en ligne : http://www.theatre-odeon.eu/fr/2014-2015/spectacles/you-aremy-destiny-lo-stupro-di-lucrezia, consulté le 24 juillet 2017. Je remercie Juliette Caron, bibliothécaire du théâtre de l'Odéon, de m'avoir aidé à identifier l'auteur de ce texte non signé.

50. Fabienne Pascaud, Télérama, art. cit.

51. Cité par Philippe Lançon, « Angélica Liddell, bourreau des mœurs » [en ligne], Libération, 15 septembre 2014, http://next.liberation.fr/theatre/2014/09/15/angelica-liddell-bourreau-des-moeurs_1101012, consulté le $1^{\text {er }}$ avril 2016.

52. Dans le texte de présentation en ligne de l'Odéon, elle est ainsi citée : «Je ne vois pas Tite-Live ou Shakespeare comme des hommes », dit-elle, « mais comme des poètes. De même que j'écris comme poète et non comme femme. »

53. «La résurrection d'Angélica Liddell », entretien avec Enric Dausset dans Théâtral magazine ${ }^{\circ}$ 50, novembre-décembre 2014, p. 41.

54. Olivier Neveux, Théâtres en lutte, Paris, La Découverte, 2007, p. 8.

55. You are my destiny, trad. par Christilla Vasserot, Besançon, Les Solitaires Intempestifs, 2014, p. 35.

56. La Maison de la force, op. cit., p. 126.

57. Ibid., p. 80.

58. Jean-Pierre Thibaudat, «You Are My Destiny: Angélica Liddell, l'amour et les hommes, waouh ! » [en ligne], Rue89, 2 décembre 2014, http://rue89.nouvelobs.com/blog/ balagan/2014/12/02/you-are-my-destiny-angelica-liddell-lamour-et-les-hommeswaouh-233805, consulté le $1^{\text {er }}$ avril 2016.

59. Annie Chénieux, "Angélica Liddell entre admiration et agacement » [en ligne], Le Journal du Dimanche, 6 décembre 2014, http://www.lejdd.fr/Culture/Theatre/ Angelica-Liddell-entre-admiration-et-agacement-704910, consulté le $1^{\text {er }}$ avril 2016.

60. Tout le ciel au-dessus de la terre, op. cit., p. 57.

61. Coline Cardi et Geneviève Pruvost, «Introduction générale ", dans Penser la violence desfemmes, op. cit., p. 13. 
Angélica Liddell sur la scène française : des limites d'une transgression « féminine »

62. Odile Quirot, «Les provocations d'Angélica Liddell », L’Obs, 27 novembre 2014, p. 118.

63. Jean-Pierre Léonardini, «Le goût amer du sacrifice »[en ligne], L'Humanité, 15 décembre 2014, http://www.humanite.fr/le-gout-amer-du-sacrifice-560187, consulté le $1^{\text {er }}$ avril 2016.

64. Annie Chénieux, Le Journal du Dimanche, art. cit.

65. Fabienne Darge, Le Monde, art. cit.

66. Fabienne Pascaud, Télérama, art. cit.

67. Jean-Pierre Léonardini, L'Humanité, art. cit. Fabienne Darge met elle aussi très vite en avant l'origine espagnole de Liddell dans son article au Monde : «Angélica L., ange et démon en blanc et noir, entre ombre et lumière, extase et enfer. Espagnole. »

68. "L'explication matérialiste de l'homme a fait place à une angoisse mystique, qui est ce qui aujourd'hui même oriente mon travail » " La explicación materialista del hombre ha dejado paso a una angustia mistica, que es lo que ahora mismo dirige mis pasos en el trabajo. »] : Christilla Vasserot cite ce propos « récent » de Liddell dans son prologue à El Sacrificio como acto poético, Madrid, Continta Me Tienes, 2014 (non traduit).

69. You are my destiny, op. cit., p. 26 : «Le temps du sacré est venu.»

70. Pour approfondir ce rapport de Liddell à la politique et à l'éthique, voir ses textes réunis dans le recueil El Sacrificio como acto poético, op. cit.

71. «[...] de partout, dans le champ théâtral, resurgissent depuis une dizaine d'années des injonctions à la politique, résurgence heureuse après la longue restauration esthétique des années 1980 - lorsque art et politique étaient sciemment désolidarisés - mais aussi suspecte par le flou qui la caractérise », Olivier Neveux, Théâtres en lutte, op. cit., p. 6. 